\title{
Circulation Journal Awards for the Year 2013
}

Hiroaki Shimokawa, MD, PhD

Dear Colleagues,

On behalf of the Editorial Team of Circulation Journal, I am pleased to announce the Circulation Journal Awards for the Year 2013.

The aim of these Awards is to recognize papers published in 2013, both clinical and experimental studies, that were highly appreciated by the Editorial Team. The selection process comprises 2 steps. In the first step, from 261 original papers published in the Journal in 2013, our 33 Japanese Associate Editors selected papers with a high scientific level in their respective fields, and in the second step, the 2 Associate Editorial Teams (16 on 1 team and 17 on the other) further evaluated the selected papers in terms of originality, contribution to cardiovascular science, manner of paper preparation, and future possibilities.

In the year of 2013, the following 4 papers have been selected for the Circulation Journal Awards.

\section{$<$ First Place in the Clinical Investigation Section >}

\section{Novel SCN3B Mutation Associated With Brugada Syndrome Affects Intracellular Trafficking and Function of Nav1.5}

Taisuke Ishikawa, Naohiko Takahashi, Seiko Ohno, Harumizu Sakurada, Kazufumi Nakamura, Young Keun On, Jeong Euy Park, Takeru Makiyama, Minoru Horie, Takuro Arimura, Naomasa Makita, Akinori Kimura

(Department of Molecular Pathogenesis, Medical Research Institute, and Division of Genetic Regulation, Graduate School of Medical and Dental Sciences, Tokyo Medical and Dental University, Tokyo; Department of Laboratory Examination and Diagnostics, Oita University, Oita; Department of Cardiovascular and Respiratory Medicine, Shiga University of Medical Science, Otsu; Department of Cardiology, Tokyo Metropolitan Hiroo Hospital, Tokyo; Department of Cardiovascular Medicine, Okayama University Graduate School of Medicine, Dentistry and Pharmaceutical Sciences, Okayama; Department of Cardiovascular Medicine, Kyoto University Graduate School of Medicine, Kyoto; Department of Molecular Pathophysiology, Nagasaki University Graduate School of Biomedical Sciences, Nagasaki, Japan; and Division of Cardiology, Samsung Medical Center, Sungkyunkwan University School of Medicine, Seoul, Korea)

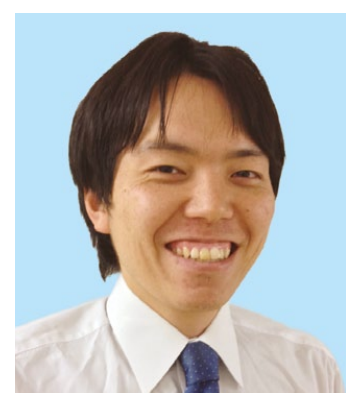

Background: Brugada syndrome $(\mathrm{BrS})$ is characterized by specific alterations on ECG in the right precordial leads and associated with ventricular arrhythmia that may manifest as syncope or sudden cardiac death. The major causes of BrS are mutations in $S C N 5 A$ for a large subunit of the sodium channel, Nav1.5, but a mutation in $S C N 3 B$ for a small subunit of sodium channel, $\operatorname{Nav} \beta 3$, has been recently reported in an American patient.

Methods and Results: A total of 181 unrelated BrS patients, 178 Japanese and 3 Koreans, who had no mutations in $S C N 5 A$, were examined for mutations in $S C N 3 B$ by direct sequencing of all exons and adjacent introns. A mutation, Val110Ile, was identified in 3 of 178 (1.7\%) Japanese patients, but was not found in 480 Japanese controls. The $S C N 3 B$ mutation impaired the cytoplasmic trafficking of Nav1.5, the cell surface expression of which was decreased in transfected cells. Whole-cell patch clamp recordings of the transfected cells revealed that the sodium currents were significantly reduced by the $S C N 3 B$ mutation.

Conclusions: The Val110Ile mutation of $S C N 3 B$ is a relatively common cause of $S C N 5 A$-negative BrS in Japan, which has a reduced sodium current because of the loss of cell surface expression of Nav1.5.1 $\quad$ (Circ J 2013; 77: 959-967) 
A

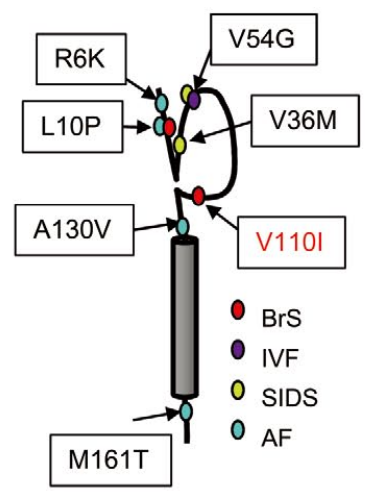

B
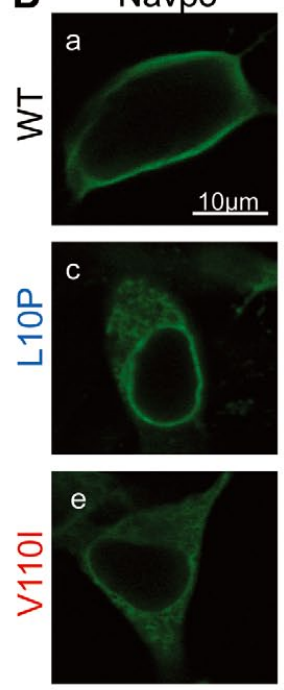

C
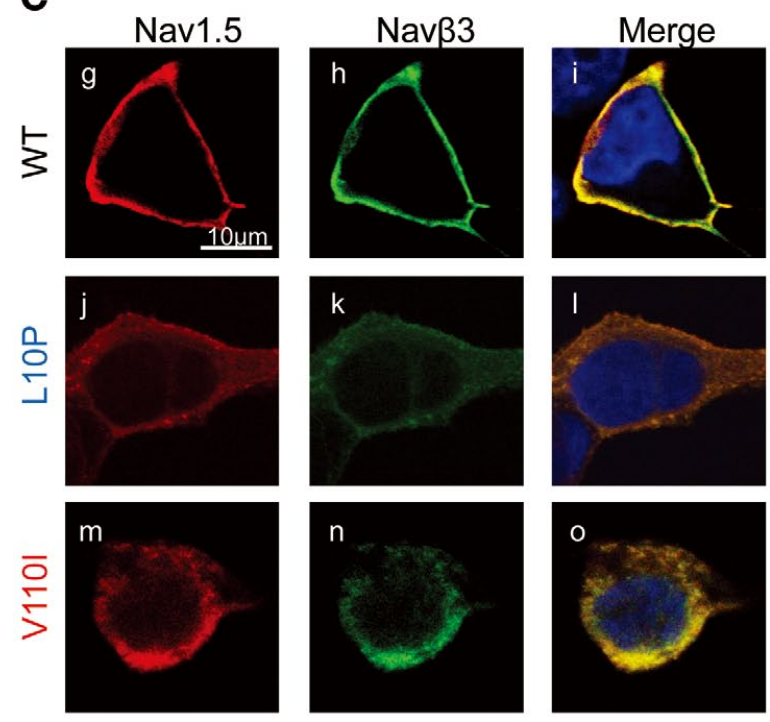

D

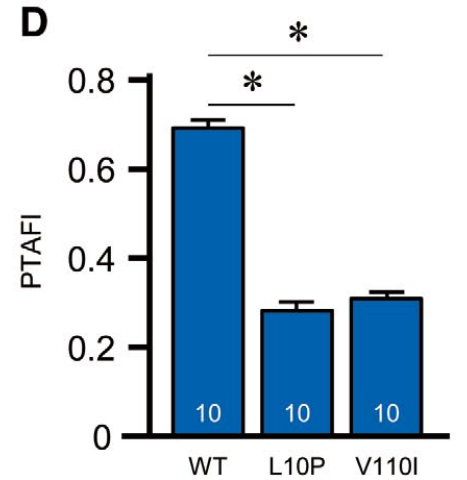

\section{Merge}
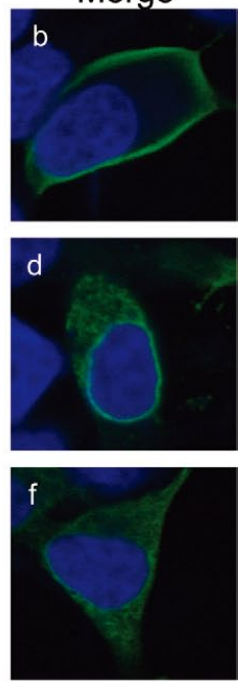

Figure 2. $S C N 3 B$ mutations reported in arrhythmias and distribution of Nav1.5 and Nav $\beta 3$ in transfected tsA-201 cells. (A) Schematic representation of $N a v \beta 3$ mutations found in arrhythmias. SCN3B mutations in BrS (red), IVF (purple), SIDS (light green), and AF (light blue) are mapped on the $\operatorname{Nav} \beta 3$. Extracellular domain and transmembrane domain are indicated. The $\mathrm{V} 110 \mathrm{l}$ mutation found in this study is indicated in red. $(\mathbf{B}, \mathbf{C})$ tsA201 cells transfected with mycHis-Nav $\beta 3-W T(\mathbf{a}, \mathbf{b})$, -L10P (c,d) or $-\mathrm{V} 110 \mathrm{O}(\mathrm{e}, \mathrm{f})$, and the combination of L1-Flag-Nav1.5 and myc-HisNavß3-WT ( $\mathbf{g}-\mathbf{i})$, -L10P (j-I), or $-\mathrm{V} 110 \mathrm{l}(\mathbf{m}-\mathbf{0})$. The cells were permeabilized by $0.15 \%$ Triton $X-100$ and stained with anti-myc $A b$ (Navß3, green; a,c,e,h,k,n) and anti-Flag Ab (Nav1.5, red; g,j,m). Nuclei were stained with DAPI and merged images are shown in b,d,f,i,l, and o. Scale bar $=10 \mu \mathrm{m}$. (D) The ratio of peripheral to total cell area fluorescence intensity (PTAFI) of expressed L1-FlaghNav1.5 in the transfected cells. Numbers of analyzed cells are indicated at the bottom of each bar. Data are expressed as mean \pm SEM. ${ }^{*} P<0.001$. AF, atrial fibrillation; BrS, Brugada syndrome; IVF, idiopathic ventricular fibrillation; SIDS, sudden infant death syndrome. 
< Second Place in the Clinical Investigation Section >

\section{Plasma Cyclophilin A Is a Novel Biomarker for Coronary Artery Disease}

Kimio Satoh, Yoshihiro Fukumoto, Koichiro Sugimura, Yutaka Miura, Tatsuo Aoki, Kotaro Nochioka, Shunsuke Tatebe, Saori Miyamichi-Yamamoto, Toru Shimizu, Shizuka Osaki, Yusuke Takagi, Ryuji Tsuburaya, Yoshitaka Ito, Yasuharu Matsumoto, Masaharu Nakayama, Morihiko Takeda, Jun Takahashi, Kenta Ito, Satoshi Yasuda, Hiroaki Shimokawa

(Department of Cardiovascular Medicine, Tohoku University Graduate School of Medicine, Sendai, Japan)

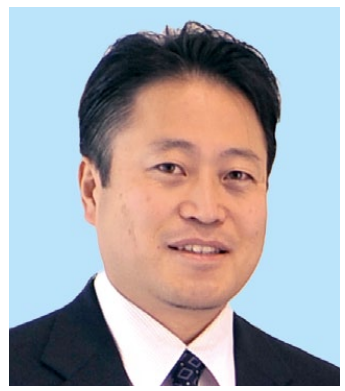

A

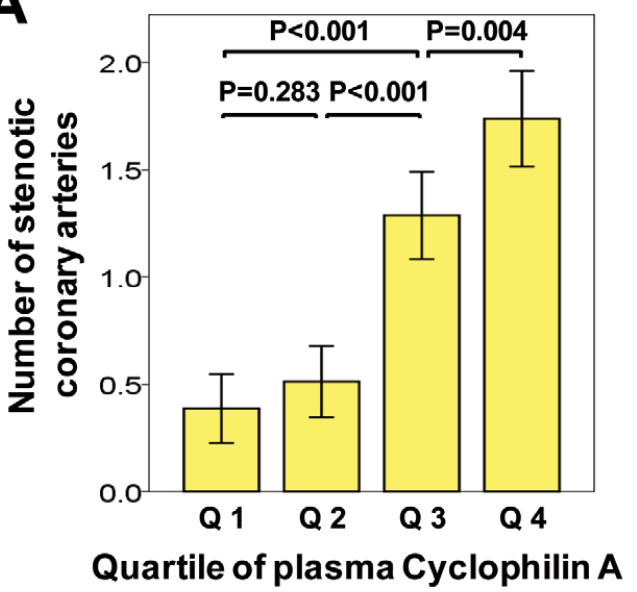

B

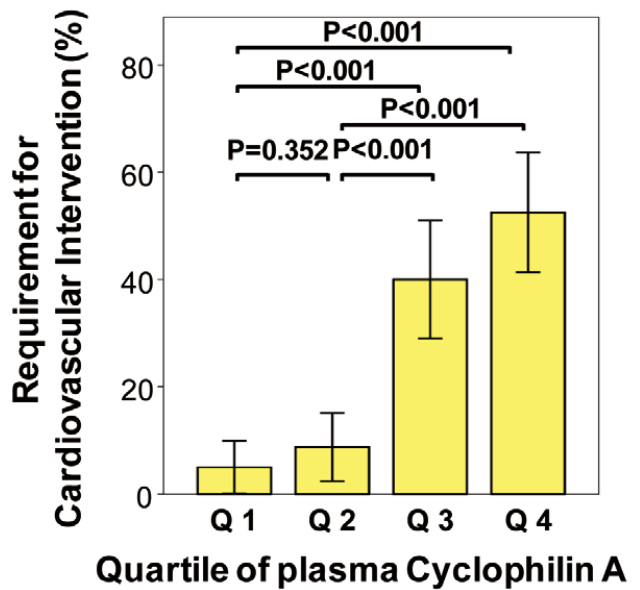

C ROC curve
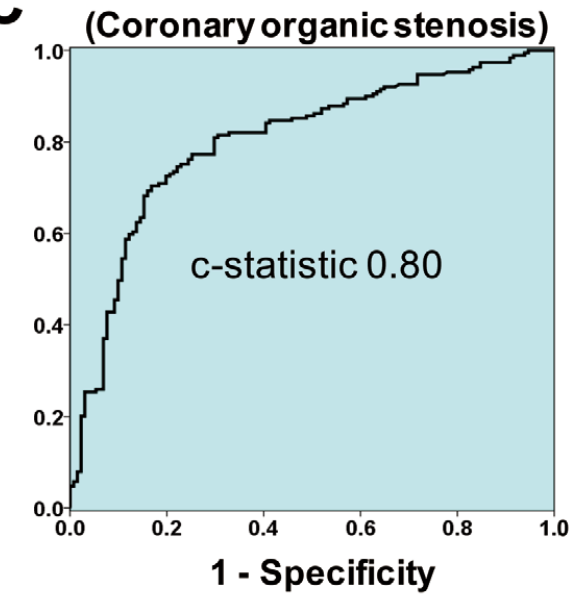

D

ROC curve

(Cardiovascular intervention)

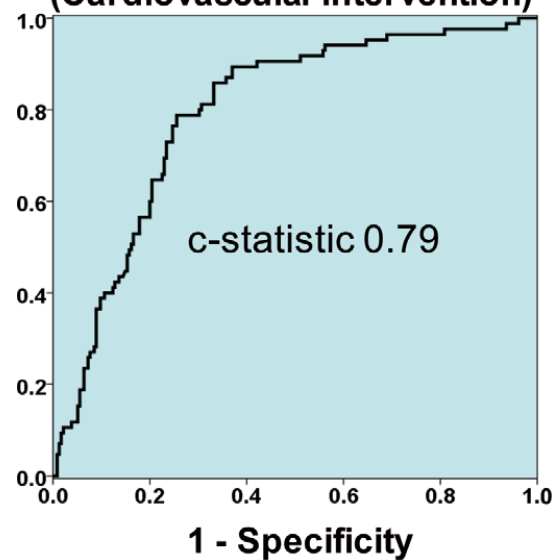

Figure 2. Number of stenotic coronary arteries and requirement for cardiovascular intervention according to quartiles of cyclophilin A (CyPA). Data from 320 patients with and without coronary stenosis are divided according to the quartiles of plasma CyPA levels. The CyPA levels were as follows: $1^{\text {st }}$ quartile (Q1), $<6.1 \mathrm{ng} / \mathrm{ml} ; 2^{\text {nd }}$ quartile (Q2), $6.2-9.6 \mathrm{ng} / \mathrm{ml} ; 3^{\text {rd }}$ quartile (Q3), $9.7-17.4 \mathrm{ng} / \mathrm{ml} ; 4^{\text {th }}$ quartile $(\mathrm{Q} 4),>17.5 \mathrm{ng} / \mathrm{ml}$. $P<0.001$ by the log-rank test for the overall comparison among the groups. (A) Number of stenotic coronary arteries according to quartiles of CyPA. CyPA was elevated in patients in Q3 $(P<0.001)$ and $Q 4 \quad(P<0.001)$ compared with $Q 1$. The number of stenotic coronary arteries increased sequentially as the quartiles increased. (B) Requirement for cardiovascular intervention according to quartiles of CyPA. The requirement was higher in patients with Q4 compared with $Q 1(P<0.001)$ and $Q 2(P<0.001)$. Cardiovascular intervention included percutaneous coronary intervention (PCl) and coronary artery bypass grafting (CABG). (C,D) Receiver-operating-characteristic curves $(\mathrm{ROC})$ and $\mathrm{c}$-statistic for baseline measurements of CyPA. Also shown are the sensitivity and specificity of these measures. (C) ROC curve describing the diagnostic performance of CyPA to identify coronary organic stenosis $>50 \%$ in at least 1 vessel as compared with the reference standard of invasive quantitative coronary angiography (CAG). The c-statistic was 0.80 (95\% Cl, 0.75-0.85). (D) ROC curve describing the diagnostic performance of CyPA to identify the requirement for future cardiovascular intervention as compared with the reference standard of invasive quantitative CAG. The c-statistic was 0.79 (95\% Cl, 0.74-0.85). Cl, confidence interval. 
Background: Oxidative stress induces secretion of cyclophilin A (CyPA) from vascular smooth muscle cells and it plays a crucial role in the pathogenesis of atherosclerosis in mice. Therefore, we tested our hypothesis that plasma CyPA levels are increased in patients with coronary artery diseases (CAD).

Methods and Results: In 320 consecutive patients undergoing coronary angiography, we examined the relationship between plasma CyPA levels and the severity of CAD. We measured plasma CyPA by an immunoassay based on the sandwich technique. Plasma CyPA levels were significantly higher in patients with significant coronary stenosis compared to those without it $(\mathrm{P}<0.001)$. A positive correlation was noted between plasma CyPA levels and significant coronary stenosis. The average number of stenotic coronary arteries and the need for coronary intervention were significantly increased in the quartiles of higher CyPA levels (both $\mathrm{P}<0.001$ ). Indeed, the plasma CyPA level significantly correlated with the presence of CAD (adjusted odds ratio for CAD, 6.20; 95\% confidence interval, 3.14-12.27; $\mathrm{P}<0.001)$. Interestingly, plasma levels of CyPA increased according to the number of atherosclerotic risk factors, all of which induce oxidative stress. Furthermore, plasma levels of CyPA significantly reduced after medical treatment of risk factors. Finally, CyPA was strongly expressed in coronary atherosclerotic plaque in patients with myocardial infarction.

Conclusions: Plasma CyPA level is a novel biomarker for oxidative stress and CAD in humans. ${ }^{2}$ (Circ J 2013; 77: 447-455)

\section{< First Place in the Experimental Investigation Section >}

\section{Ultrastructural Maturation of Human-Induced Pluripotent Stem Cell- Derived Cardiomyocytes in a Long-Term Culture}

Tsukasa Kamakura, Takeru Makiyama, Kenichi Sasaki, Yoshinori Yoshida, Yimin Wuriyanghai, Jiarong Chen, Tetsuhisa Hattori, Seiko Ohno, Toru Kita, Minoru Horie, Shinya Yamanaka, Takeshi Kimura

(Department of Cardiovascular Medicine, Kyoto University Graduate School of Medicine, Kyoto; Department of Cardiovascular and Respiratory Medicine, Shiga University of Medical Science, Otsu; Center for iPS Cell Research and Application (CiRA), Institute for Integrated Cell-Material Sciences Kyoto University, Kyoto; and Department of Cardiovascular Medicine, Kobe City General Hospital, Kobe, Japan)

Background: In the short- to mid-term, cardiomyocytes generated from human-induced pluripotent stem cells (hiPSC$\mathrm{CMs}$ ) have been reported to be less mature than those of adult hearts. However, the maturation process in a long-term culture remains unknown.

Methods and Results: A hiPSC clone generated from a healthy control was differentiated into CMs through embryoid body (EB) formation. The ultrastructural characteristics and gene expressions of spontaneously contracting EBs were analyzed through 1-year of culture after cardiac differentiation was initiated. The 14-day-old EBs contained a low number of myofibrils, which lacked alignment, and immature high-density Z-bands lacking A-, H-, I-, and M-bands. Through the long-term culture up to 180 days, the myofibrils became more tightly packed and formed parallel arrays accompanied by the appearance of mature Z-, A-, H-, and I-bands, but not M-bands. Notably, M-bands were finally detected in 360-day-old EBs. The expression levels of the M-band-specific genes in hiPSC-CMs remained lower in comparison with those in the adult heart. Immunocytochemistry indicated increasing number of MLC2v-positive/ MLC2a-negative cells with decreasing number of MLC2v/MLC2a double-positive cells, indicating maturing of ventricular-type CMs.

Conclusions: The structural maturation process of hiPSC-CMs through 1-year of culture revealed ultrastructural sarcomeric changes accompanied by delayed formation of M-bands. Our study provides new insight into the maturation process of hiPSC-CMs. ${ }^{3}$ (Circ J 2013; 77: 1307-1314) 


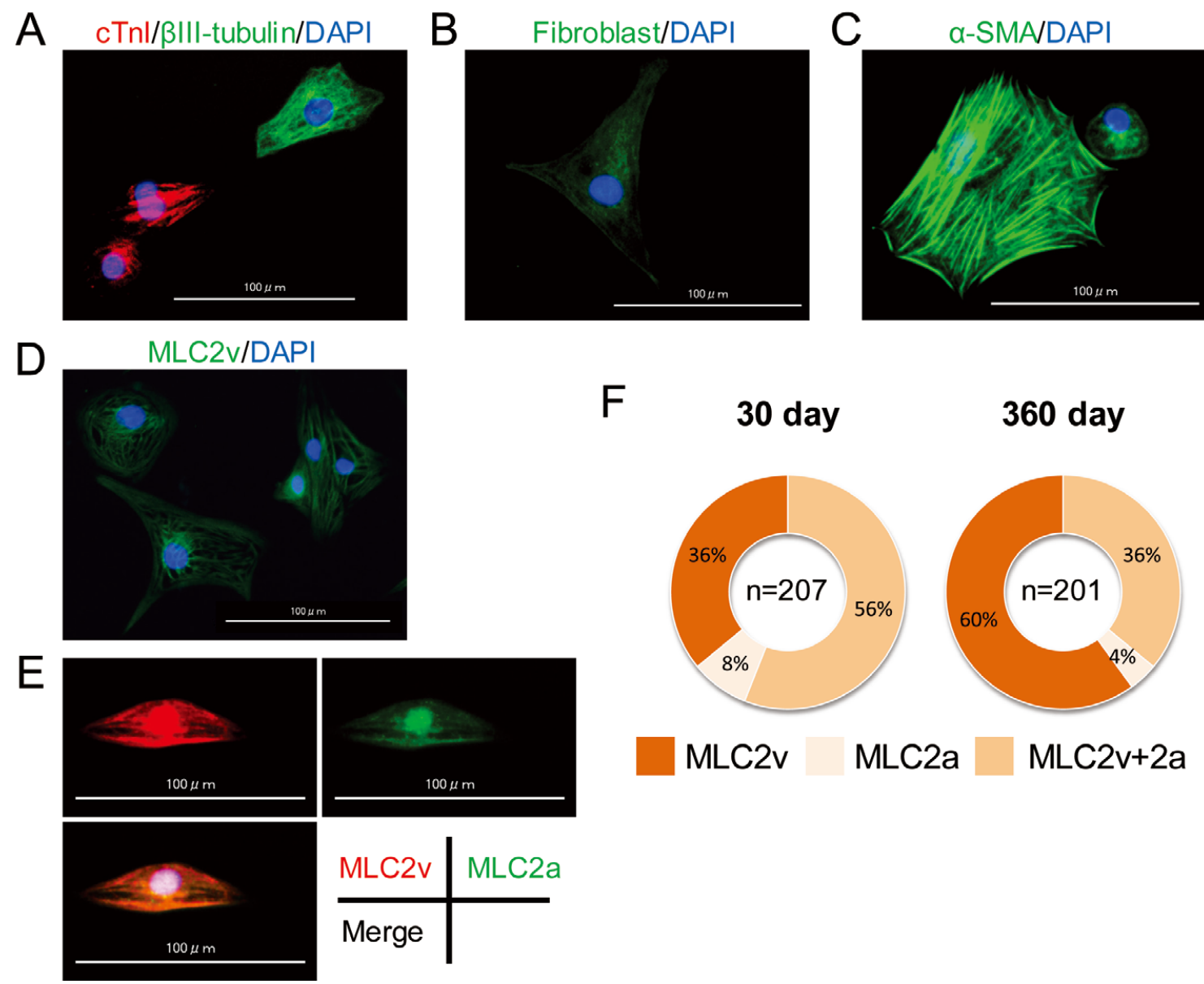

Figure 2. Immunostaining of human-induced pluripotent stem cell-derived cardiomyocytes (hiPSC-CMs) at 30 days after cardiac differentiation. (A-E) Immunostaining of single cells isolated from microdissected beating embryoid bodies (EBs) at 30 days using the following antibodies: (A) CTnl (red), $\beta$ III-tublin (green), and DAPI (blue); (B) fibroblast (green) and DAPI (blue); (C) a-SMA (green) and DAPI (blue); (D) MLC2v (green) and DAPI (blue); (E) MLC2v (red) and MLC2a (green). (E) hiPSC-CM expressing both MLC2v and MLC2a. (F) Properties of MLC2v-positive/MLC2a-negative, MLC2v-negative/MLC2apositive, and MLC2v/MLC2a double-positive cells at 30 days and 360 days. Among the MLC2v- or MLC2a-positive cells, $36 \%$ of 30-day hiPSC-CMs were MLC2v-positive/MLC2a-negative mature ventricular CMs and 56\% were MLC2v/MLC2a double-positive immature ventricular CMs. At day 360, MLC2v-positive/MLC2a-negative mature CMs increased to 60\%, whereas MLC2v/MLC2a double-positive immature ventricular CMs decreased to $36 \%$.

< Second Place in the Experimental Investigation Section >

\section{Myocardial Layer-Specific Effect of Myoblast Cell-Sheet Implantation Evaluated by Tissue Strain Imaging}

Yasuhiro Shudo, Shigeru Miyagawa, Satoshi Nakatani, Satsuki Fukushima, Taichi Sakaguchi, Atsuhiro Saito, Toshihiko Asanuma, Naomasa Kawaguchi, Nariaki Matsuura, Tatsuya Shimizu, Teruo Okano, Yoshiki Sawa

(Department of Cardiovascular Surgery, Department of Health Sciences, Division of Functional Diagnostics, Department of Pathology, Osaka University Graduate School of Medicine, Suita; and Advanced Biomedical Engineer and Science, Tokyo Women's Medical University, Tokyo, Japan)

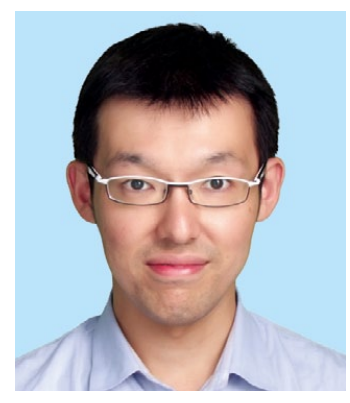


Background: The implantation of skeletal myoblast (SMB) cell-sheets over the damaged area of a myocardial infarction (MI) has been shown to improve global left ventricular (LV) function through a paracrine effect. However, the regeneration process has not been fully evaluated. We hypothesized that the use of tissue Doppler strain M-mode imaging to assess myocardial layer-specific strain might enable detailed visual evaluation of the regenerative ability of SMBs.

Methods and Results: SMBs were cultured on temperature-responsive culture dishes to generate cell-sheets. At 4 weeks after inducing anterior MI, the animals were divided into 2 groups: SMB cell-sheet implantation and sham operation ( $n=6$ in each). A total of 30 cell-sheets $\left(1.5 \times 10^{7}\right.$ cells/sheet $)$ were placed on the epicardium, covering the infarct and border regions. Subendocardial and subepicardial strain values were measured in the infarct, border, and remote regions by tissue Doppler strain analysis. SMB cell-sheet implantation produced the following major effects: progression of LV remodeling was prevented and global LV ejection fraction increased; the subendocardial strain was significantly greater than the subepicardial strain in the treated border region; vascular density in the subendocardium was significantly higher than in the subepicardium in the treated region; the expression of vascular endothelial growth factor was significantly increased.

Conclusions: Tissue Doppler strain analysis allows precise evaluation of the effect of cell-sheet implantation on layer-specific myocardial function. ${ }^{4} \quad$ (Circ J 2013; 77: 1063-1072)

\section{Infarcted area $(\%)$}

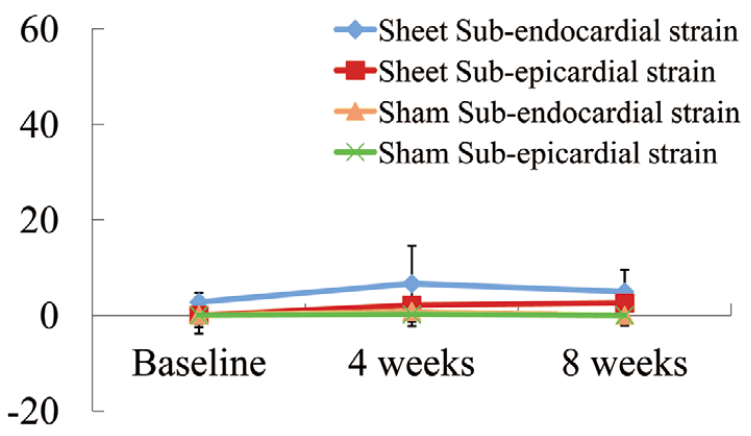

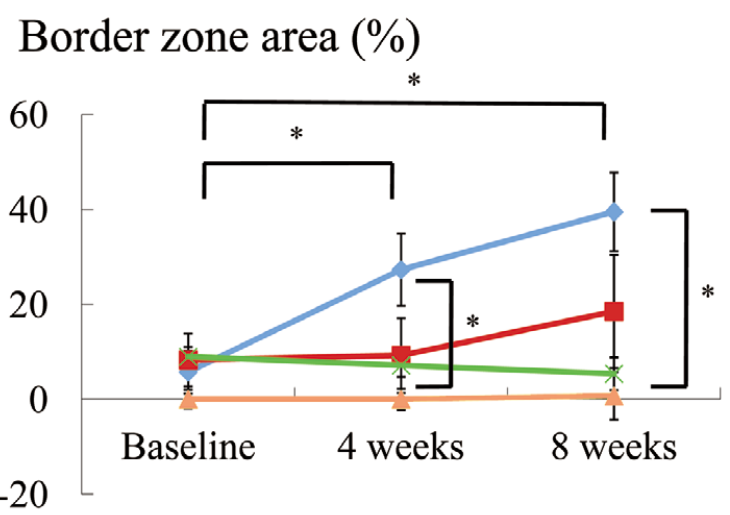

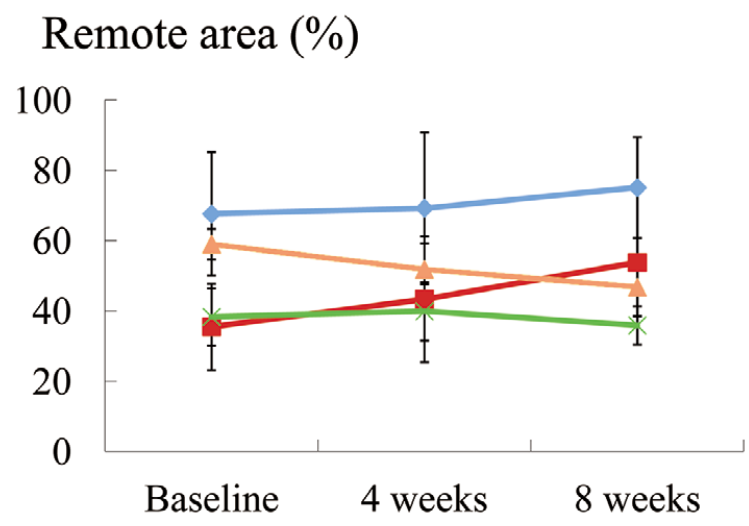

Figure 4. Myocardial layer strain value. In the treated border region, subendocardial strain significantly increased at 4 and 8 weeks after cell-sheet implantation, showing a significant increase in comparison with subepicardial strain. In the infarcted region, both subendocardial and subepicardial strain values were greater in the Sheet group than in the Sham group. In the remote region, no significant changes were observed. $n=6$ in each group; ${ }^{*} \mathrm{P}<0.05$. 
Awards will be presented to the 4 research groups during the 78th Annual Scientific Meeting of the Japanese Circulation Society, and will also be announced on the Society website. We look forward to receiving manuscripts with high scientific impact for publication in Circulation Journal in 2014.

Hiroaki Shimokawa, MD, PhD

Editor-in-Chief

Circulation Journal

(Released online February 7, 2014)

\section{References:}

1. Ishikawa T, Takahashi N, Ohno S, Sakurada H, Nakamura K, On YK, et al. Novel SCN3B mutation associated with brugada syndrome affects intracellular trafficking and function of Nav1.5. Circ J 2013; 77: 959-967.

2. Satoh K, Fukumoto Y, Sugimura K, Miura Y, Aoki T, Nochioka K, et al. Plasma cyclophilin A is a novel biomarker for coronary artery disease. Circ J 2013; 77: 447-455.

3. Kamakura T, Makiyama T, Sasaki K, Yoshida Y, Wuriyanghai Y, Chen J, et al. Ultrastructural maturation of human-induced pluripotent stem cell-derived cardiomyocytes in a long-term culture. Circ J 2013; 77: 1307-1314.

4. Shudo Y, Miyagawa S, Nakatani S, Fukushima S, Sakaguchi T, Saito A, et al. Myocardial layer-specific effect of myoblast cell-sheet implantation evaluated by tissue strain imaging. Circ J 2013; 77: 1063-1072. 